\title{
Domain-shift Conditioning using Adaptable Filtering via Hierarchical Embeddings for Robust Chinese Spell Check
}

\author{
Minh Nguyen Student Member, IEEE, Gia H. Ngo, and Nancy F. Chen Senior Member, IEEE
}

\begin{abstract}
Spell check is a useful application which processes noisy human-generated text. Spell check for Chinese poses unresolved problems due to the large number of characters, the sparse distribution of errors, and the dearth of resources with sufficient coverage of heterogeneous and shifting error domains. For Chinese spell check, filtering using confusion sets narrows the search space and makes finding corrections easier. However, most, if not all, confusion sets used to date are fixed and thus do not include new, shifting error domains. We propose a scalable adaptable filter that exploits hierarchical character embeddings to (1) obviate the need to handcraft confusion sets, and (2) resolve sparsity problems related to infrequent errors. Our approach compares favorably with competitive baselines and obtains SOTA results on the 2014 and 2015 Chinese Spelling Check Bake-off datasets.
\end{abstract}

\section{INTRODUCTION}

$\mathbf{S}$ PELL check is a common task in processing written text, as spell checkers are an integral component in text editors and search engines. A spell checker must identify erroneous words/characters and suggest candidates for correction (Figure 1). Despite its utility, spell check for Chinese presents unresolved challenges stemming from the language's numerous characters (up to 10k), the sparsity of errors, and the scarcity of linguistic resources with sufficient coverage of heterogeneous and shifting error domains.

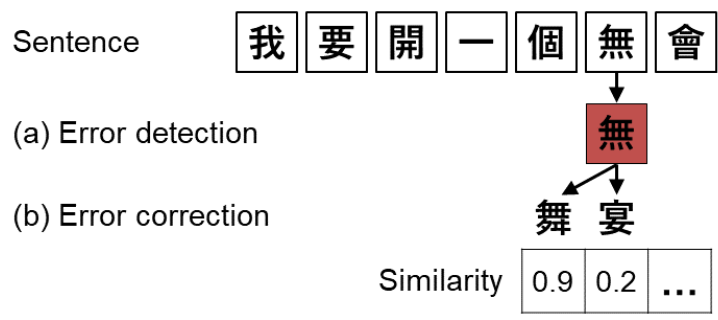

Fig. 1. Spell check for Chinese (a) Error detection (b) Error correction (suggesting correction candidates)

\section{A. Background}

Spell check is more challenging for Chinese than for languages like English as there are more options (characters) to

(C) 2021 IEEE. Personal use of this material is permitted. Permission from IEEE must be obtained for all other uses, in any current or future media, including reprinting/republishing this material for advertising or promotional purposes, creating new collective works, for resale or redistribution to servers or lists, or reuse of any copyrighted component of this work in other works. Nancy F. Chen is the corresponding author (nancychen@alum.mit.edu). correct an erroneous character. There are up to $10 \mathrm{k}$ common Chinese characters compared to only 26 characters for English. The sparse and shifting errors also make training a good spell checker challenging. Errors belong to different domains depending on whether the text with errors is typed or handwritten. User demographics and degree of formality may also shift the domain of errors. For example, a spell checker trained using errors collected from essays hand-written by second language learners for academic exams may not generalize well to text typed by native language users on social media platforms.

(a) Error Histogram Count

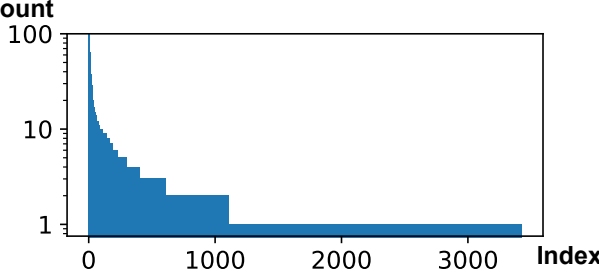

(b) Error Overlap

(c) Error Coverage
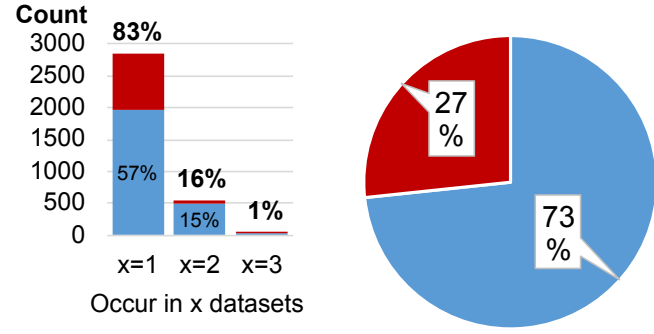

- In CFS - Not in CFS

Fig. 2. Characterizing error domains in datasets from 3 consecutive CSC Bake-offs (a) Error sparsity; More than $2 k$ types of error occur only once across 3 years. (b) Domain-shift from year to year; 83\% of types of error do not overlap across 3 years. (c) Domain-shift reduces coverage of given confusion sets (CFS) to only $73 \%$.

Figure 2 illustrates the challenges from three perspectives using datasets from 3 consecutive years of the Chinese spell check (CSC) Bake-offs [1-3]. Figure 2a shows the histogram of error types in the 3 training sets, in which there is a long tail with more than 2000 error types that occur only once (error sparsity). Figure $2 \mathrm{p}$ shows the numbers of error types that occur in 1, 2, or all 3 datasets. The error domains differ vastly between datasets (i.e. domain-shift [4,5]) with only $1 \%$ of error types common across all 3 datasets. Most types of 
error occur exclusively in one dataset (83\%).

Confusion sets [6] are often used to tackle error sparsity. The confusion set of a character consists of phonologically or morphologically similar characters. For example, the confusion set of 無 can be $\{$ 吾,嫵,舞\}. Due to their similarity, 無 can be mistaken for any character in its confusion set. As confusion sets define characters that are likely substitution errors, they can be used to filter out unlikely corrections even for characters unseen in the training data.

However, confusion sets [1] are often handcrafted and may be susceptible to domain-shift. As error domains in the data diverge from error domains captured by the handcrafted confusion sets, the number of errors covered by the confusion sets drop to $73 \%$, leaving a quarter of the errors at risk of mis-detection (Figure 22). The reduced coverage may also limit the ability to detect infrequent spelling errors, which is in reality a sizable amount of the errors (more than $60 \%$ of errors only occur once), given the extremely long tail in the error distribution (see Figure 2).

Constructing confusion sets covering all error domains is nontrivial because there are numerous different domains with various error patterns. Besides the difference between typed and hand-written text, even within typed text, error patterns differ depending on the input method (IME) used. Using phonetic-based IMEs like Sogou Pinyin requires inputting pronunciation, so errors often are caused by pronunciation similarity. In contrast, using morphology-based IMEs like Wubi requires inputting characters' components, so errors are often caused by morphological similarity. Covering multiple domains is required for accurate spell check because even the same user may commit errors in different domains when using different IMEs (e.g. a user who typically uses morphologybased IMEs might switch to phonetic-based IMEs when she forgets how to write but knows how to pronounce a Chinese character).

Keeping the confusion sets up-to-date is as difficult as constructing them as error patterns evolve or new domains emerge. There is much work attempting to expand or construct confusion sets to increase coverage [7- 7 ]. However, these methods involve human experts handcrafting the function that measures similarity between characters [9] or handcrafting how similar characters are gathered [7, 8]. It is difficult to scale methods with handcrafted rules to capture shifting error domains.

\section{B. Proposed Solution}

Due to the sparsity of spelling errors (many errors observed only once in the training data, or possibly not at all) and the large number of Chinese characters, training a spell check model that directly predicts spell error corrections is difficult. We propose a spell check model with an adaptable filter that refines its prediction by considering phonological and morphological similarity between characters. The adaptable filter is constructed using a similarity function learned from data using hierarchical character embeddings [10]. Since similarity between characters is one of the primary factors causing substitution errors [11], the refinement would make spell check more accurate. Although character embeddings have been used as model input in prior work [10,12], as far as we know, our work is the first that uses character embeddings to filter model output to improve accuracy. Unlike handcrafted confusion sets, our filter can be trained using errors in the training data so it is less affected by domain-shift. Experimental results show that the proposed model with adaptive filtering is more accurate than baseline models, obtaining SOTA results on the 2014 and 2015 Chinese Spelling Check Bake-off datasets.

\section{RELATED WORK}

Spell check involves error detection and correction to which there are several approaches. Error detection was tackled using language model [13-15], conditional random field [16, 17], graph-based algorithm [18-21], or sequence tagging model [22-24].

Most approaches to error correction [25, 26] share two common points. First, they use confusion sets [27, 28] to filter out unlikely correction candidates or to generate candidates for beam-search decoding [29]. The confusion sets are often constructed once using hand-crafted similarity functions [30] and stay fixed thereafter. Second, most approaches involve data augmentation [31,32] or transfer learning [24, 33, 34] to compensate for the limited size of CSC training sets.

Our work is similar to [24, 33, 34], although our filtering is different. Xie et al. [24] used fixed filtering while our filtering is adaptable. Hong et al. [33] filtering can be fine-tuned albeit manually while our filter can be fine-tuned automatically using training data. While Cheng et al. [34]'s filtering exploits only similarity between characters, our approach also exploits character structural similarity by using hierarchical embeddings. Our filter adaptation is a form of supervised domain adaptation [35] in which labeled errors help capture additional error domains that are missed by the given confusion sets. Although domain adaptation is usually framed as semi-supervised learning where target domain examples are unlabeled [36, 37], semi-supervised adaptation has yet to outperform supervised adaptation in terms of performance improvement [38-40]. Thus, through adaptation, our approach may generalize to more error domains and scale more elegantly since it does not require manual tuning.

\section{APPROACH}

The model consists of a pre-trained masked language model (BERT [41]) and our proposed Hierarchical Embedding Adaptable filter (HeadFilt). Leveraging pre-trained language model (LM) has led to successes in numerous NLP tasks [4245]. However, predicting corrections using only the masked LM (Figure 3a) is challenging due to inadequate training data and the large number of Chinese characters. To boost accuracy, LM's predictions are filtered using HeadFilt (Figure 3 b).

Let the input sequence be $\mathbf{X}_{*}$, where $X_{i}$ denotes the $i$-th character in the sequence. All unique characters across all sequences belong to the vocabulary $\{X\}$. Given $\mathbf{X}_{*}$, the masked LM outputs $\tilde{\mathbf{Y}}_{*}$, whereby $\tilde{\mathbf{Y}}_{i}=\left\{\tilde{Y}_{i k}\right\}$ is the distribution over corrections at $i$. The predicted correct character at $i$ before filtering is $\tilde{Z}_{i}$ (Equation 2). 


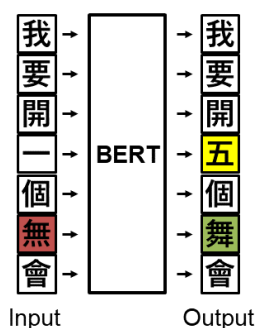

(a) Without filtering

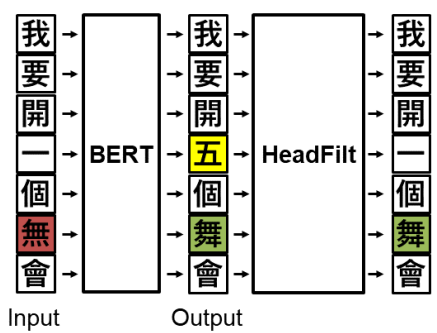

(b) With filtering True positive: 無 $\rightarrow$ 舞

Fig. 3. Advantage of filtering (a) Prediction without filtering. (b) Prediction with filtering. BERT's mistake is corrected by filtering. Hierarchical Embedding Adaptable Filter (HeadFilt) is described in Section III-B. Red: error, Green: correction, Yellow: false positive.

For each input character $X_{i}$, HeadFilt computes a similarity vector of size $N, \mathbf{S}_{i}$, which is the similarity between $X_{i}$ and the set of all $N$ characters in the vocabulary (i.e. $\{X\}$ ). HeadFilt is described in Section III-A The similarity vector $\mathbf{S}_{i}$ is element-wise multiplied with $\mathbf{Y}_{i}$ to filter out unlikely corrections. The predicted correct character at position $i$ after filtering is $\tilde{Z}_{i}^{\text {filt }}$ (Equation 4).

$$
\begin{aligned}
\tilde{\mathbf{Y}}_{*} & =\operatorname{MaskLM}\left(\mathbf{X}_{*}\right) \\
\tilde{Z}_{i} & =\underset{k}{\arg \max } \tilde{Y}_{i k} \\
\mathbf{S}_{i} & =\operatorname{HeadFilt}\left(X_{i} ;\{X\}\right) \\
\tilde{Z}_{i}^{\text {filt }} & =\underset{k}{\arg \max }\left(\tilde{\mathbf{Y}}_{i} \odot \mathbf{S}_{i}\right)_{k}
\end{aligned}
$$

\section{A. Adaptable Filter with Hierarchical Embeddings}

Filtering out unlikely candidates using confusion sets can significantly boost accuracy [27, 28, 30]. However, under domain-shift, fixed confusion sets could miss errors from new domains. We propose an adaptable filtering model named HeadFilt which can be fine-tuned using errors from new domains to alleviate the domain-shift problem. Filter fine-tuning is a form of supervised domain adaptation as it minimizes the domain-shift between errors captured by the given confusion sets and the errors in the training data. HeadFilt estimates likelihood of correction candidates using distances in the embedding space between characters. HeadFilt uses hierarchical character embeddings [10] which have inductive bias of the character structure so as to extend filtering to phonologically and morphologically characters unseen in the training data, thus addressing the error sparsity problem. Hierarchical character embedding is obtained by applying TreeLSTM [46, 47] on the tree structure of the character. Figure 4 shows the tree structure of a character and its hierarchical embedding $\left(\mathbf{h}_{\mathbf{7}}\right)$.

A (fixed) confusion set of a character can be represented as a binary similarity vector ( $\mathbf{S}$ in Figure $5 \mathrm{k}$ ) with a 1 for each character in the set that is deemed possibly confused with the given character. In contrast, the similarity vector $(\widehat{\mathbf{S}}$ in Figure $5 \mathrm{k}$ ) produced by HeadFilt has real-valued scores estimated using the structure of the characters. Combining all the confusion sets (i.e. concatenating all the binary vectors) results in a similarity matrix (Figure 5 p).

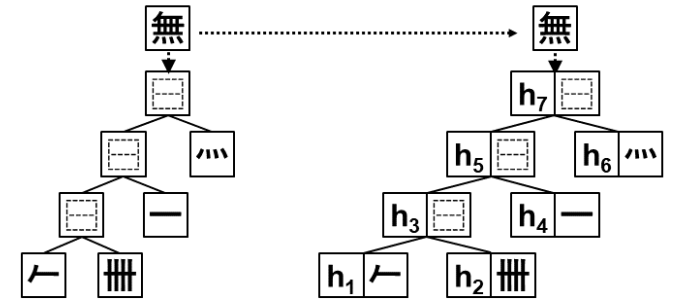

(a) Structure

(b) Embedding

Fig. 4. Hierarchical embedding (a) Character as a tree with sub-character components as leaves. Internal nodes denote the relative spatial positions of the character components. (b) Hierarchical embedding $\left(\mathbf{h}_{\mathbf{7}}\right)$ constructed from tree (a)

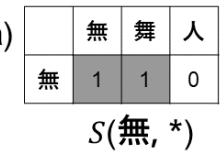

(b)

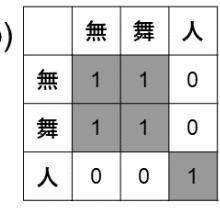

(c)

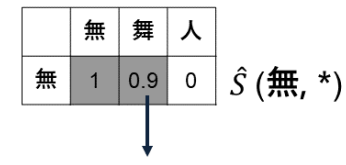

Similarity Score

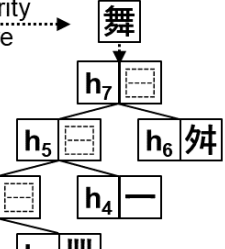

Fig. 5. (a) Similarity vector constructed from the confusion set of character 無 (b) All confusion sets (c) Similarity vector constructed by HeadFilt

Let the hierarchical embeddings of character $a$ and $b$ be $\mathbf{h}_{\mathbf{a}}$ and $\mathbf{h}_{\mathbf{b}}$. The HeadFilt similarity between $a$ and $b, \widehat{S}(a, b)$, is estimated using Equation 6 The constants $\beta$ and $m$ in Equation 6 are the scaling factor and the margin respectively. Intuitively, $\widehat{S}(a, b)$ in Equation 6 is close to 1 if the L2 distance $\left(d_{a b}\right)$ between the two embedding vectors is smaller than the margin $m$ and is close to 0 otherwise. Let $\widehat{\mathbf{S}}_{i}$ be the HeadFilt similarity vector of the character $X_{i}$. Vector $\widehat{\mathbf{S}}_{i}$ is the concatenation of similarity scores of $X_{i}$ and all other $N$ characters $\left(c_{1}, c_{2}, \ldots c_{N}\right)$ in the vocabulary. (Equation 7 ).

$$
\begin{aligned}
d_{a b} & =\left\|\mathbf{h}_{\mathbf{a}} /\right\| \mathbf{h}_{\mathbf{a}}\left\|-\mathbf{h}_{\mathbf{b}} /\right\| \mathbf{h}_{\mathbf{b}}\|\| \\
\widehat{S}(a, b) & =\frac{1}{1+\exp \left(\beta \times\left(d_{a b}-m\right)\right)} \\
\widehat{\mathbf{S}}_{i} & =\left[\widehat{S}\left(X_{i}, c_{1}\right), \ldots \widehat{S}\left(X_{i}, c_{N}\right)\right]
\end{aligned}
$$

In addition, let $\mathbf{S}_{i}$ be the confusion set similarity vector of $X_{i}$. The prediction after filtering $\left(\tilde{Z}_{i}^{\text {filt }}\right)$ is:

$$
\begin{aligned}
& \tilde{\mathbf{Y}}_{i}^{\text {filt }}=\tilde{\mathbf{Y}}_{i} \odot \mathbf{S}_{i} \text { (using fixed confusion set) } \\
& \tilde{\mathbf{Y}}_{i}^{\text {filt }}=\tilde{\mathbf{Y}}_{i} \odot \widehat{\mathbf{S}}_{i} \quad \text { (using HeadFilt) }
\end{aligned}
$$

\section{B. Adaptable Filter Training}

HeadFilt is trained by minimizing the contrastive loss [48] in Equation 10 below. Minimizing the loss forces L2 distances between similar characters to be within the margin $m$ and L2 distances between dissimilar characters to be greater than 
the margin $m$. Let $S(a, b)$ be the observed similarity of two characters $a$ and $b$.

$$
\begin{aligned}
L_{c} & =\sum_{a, b}\left[S(a, b) \times \max \left(0, d_{a b}-m\right)\right. \\
& \left.+(1-S(a, b)) \times \max \left(0, m-d_{a b}\right)\right]
\end{aligned}
$$

There are two sources of examples for training the filter: i.e. the positive and negative examples from the confusion sets and the positive examples observed in the training data. A positive example is an error pair $(a, b)$ where $a$ is mistaken for $b$ or vice-versa. Thus, all pair $(a, b)$ in the same confusion set and all errors $(a, b)$ in the training data are positive examples. Other character pairs are considered as negative examples. For positive examples, $a$ and $b$ are similar and $S(a, b)=1$, while for negative examples, $a$ and $b$ are dissimilar and $S(a, b)=0$.

The objective of the optimization in Equation 10 is to ensure that (1) the distances between similar characters are smaller than the margin $m$ and (2) the distances between dissimilar characters are larger than the margin $m$. When a and $\mathrm{b}$ are similar, $S(a, b)=1$, the loss contributed by $a$ and $b$ is $\max \left(0, d_{a b}-m\right)$. Minimizing the loss will push the embeddings of $a$ and $b$ closer to one another if only $d_{a b}>m$. When a and $\mathrm{b}$ are dissimilar, $S(a, b)=0$, the loss contributed by $a$ and $b$ is $\max \left(0, m-d_{a b}\right)$. Minimizing the loss will push the embeddings of $a$ and $b$ further apart if only $d_{a b}<m$.

HeadFilt is trained in two steps: (1) imitating the given confusion sets, where characters in the same confusion sets are cast as positive examples and characters not in the same confusion sets are cast as negative examples. (2) domainshift conditioning (adaptation), where error pairs observed in the training data are further added as positive examples and the filter is trained further using this larger set of examples. Combining examples from both source and target domains for supervised adaptation was also done in [35]. The effect of domain adaptation is shown in Section IV-F

To calculate $\widehat{S}(a, b)$ (Equation 6 ), we need the values of $m$ and $\beta$. In our experiments, we set $m=0.4$. Besides, we need to set $\beta$ so that when $a$ and $b$ are dissimilar, the probability of predicting $b$ as the correction for $a$ or vice-versa is very small (less than chance) as shown in Equation 11 .

$$
\begin{aligned}
& \frac{1}{1+\exp \left(\beta \times\left(d_{a b}-m\right)\right)} \leq \frac{1}{N}, \\
& \forall a, b \text { s.t. } S(a, b)=0 \\
& \beta \geq \frac{\ln (N-1)}{d_{*}-m} \\
& d_{*}=\frac{\sum_{a, b}(1-S(a, b)) * d_{a b}}{\sum_{a, b}(1-S(a, b))}
\end{aligned}
$$

However, solving Equation 11 for all pairs $(a, b)$ (including unobserved pairs) is intractable. Thus, we approximate Equation 11 by Equation 12 using positive and negative examples in the training data. Setting $\beta$ according to Equation 12 ensures that on average when $a$ and $b$ are dissimilar, it is very unlikely to predict $b$ as the correction for $a$.

\section{EXPERIMENTS}

\section{A. Data}

We used datasets from the 2013 [1], 2014 [2], and 2015 [3] Chinese Spell Check Bake-offs. For each dataset, we only used its training set to fine-tune the models (BERT and HeadFilt). Table I shows the statistics of the datasets. Unlike 2014 and 2015, there are two test sets in 2013: one for error detection and one for error correction.

\begin{tabular}{llr} 
Dataset & With Error & Length \\
\hline 2013 Train & $350 / 700$ & 41.8 \\
2013 Test (Detection) & $300 / 1000$ & 68.7 \\
2013 Test (Correction) & $996 / 1000$ & 74.3 \\
\hline 2014 Train & $3432 / 3435$ & 49.6 \\
2014 Test & $529 / 1062$ & 50.0 \\
\hline 2015 Train & $2339 / 2339$ & 31.3 \\
2015 Test & $550 / 1100$ & 30.6 \\
\multicolumn{3}{c}{ Amount of data (sentences) in the datasets. }
\end{tabular}

We used the confusion sets [11] provided by the 2013 Bake-off for filtering in our baseline model and for training the HeadFilt model. As the confusion sets were created in 2011, it may not cover newer error domains (i.e. domainshift). Table $\Pi$ II shows the number of errors in the training data

Training set Errors covered by confusion sets

\begin{tabular}{lr}
\hline 2013 & $252 / 269(93.68 \%)$ \\
2014 & $1641 / 2197(74.69 \%)$ \\
2015 & $1177 / 1568(75.06 \%)$
\end{tabular}

TABLE II

Number of unique errors in the training sets that are covered by the given confusion sets.

that are covered by the confusion sets. Although coverage is above $90 \%$ for 2013 , it drops by $20 \%$ absolute for 2014 and 2015. This drop could conceivably be due to different data collection methods. While the 2013 dataset was from written essays by first language learners of Chinese, the 2014 and 2015 datasets were from typed essays by second language learners of Chinese. Despite the lack of coverage, HeadFilt can adapt to errors in the training sets to filter better.

\section{B. Baselines}

The proposed approach has two stages in which the masked LM's output from the first stage is filtered by HeadFilt in the second stage. We compared the proposed approach against 3 baselines:

1. BERT which does not filter predictions.

2. FixedFilt which uses the provided fixed confusion sets for filtering.

3. GlyceFilt which uses Glyce embeddings [12] instead of hierarchical embeddings.

Although there are many other embedding models for Chinese characters [49-55], we chose the Glyce embedding model as it 


\begin{tabular}{|c|c|c|c|c|}
\hline Prediction & Acc. & Pre. & Rec. & F1 \\
\hline \multicolumn{5}{|c|}{2013 Bake-off } \\
\hline SMT & 0.861 & 0.845 & 0.656 & 0.739 \\
\hline n-gram LM & 0.825 & 0.745 & 0.633 & 0.684 \\
\hline FASpell & 0.631 & 0.762 & 0.632 & 0.691 \\
\hline BERT & 0.905 & 0.835 & 0.851 & 0.842 \\
\hline FixedFilt & 0.910 & 0.884 & 0.804 & 0.842 \\
\hline GlyceFilt & 0.917 & 0.910 & 0.804 & 0.854 \\
\hline HeadFilt & 0.914 & 0.891 & 0.813 & 0.850 \\
\hline \multicolumn{5}{|c|}{2014 Bake-off } \\
\hline KUAS & 0.719 & 0.914 & 0.484 & 0.633 \\
\hline FASpell & 0.700 & 0.610 & 0.535 & 0.570 \\
\hline SpellGCN $\dagger$ & - & 0.583 & 0.545 & 0.563 \\
\hline MERT & 0.700 & 0.787 & 0.548 & 0.646 \\
\hline BERT & 0.708 & 0.780 & 0.580 & 0.665 \\
\hline FixedFilt & 0.709 & 0.831 & 0.526 & 0.644 \\
\hline GlyceFilt & 0.702 & 0.824 & 0.515 & 0.633 \\
\hline HeadFilt & 0.719 & 0.823 & 0.559 & 0.666 \\
\hline \multicolumn{5}{|c|}{2015 Bake-off } \\
\hline HanSpeller & 0.700 & 0.802 & 0.532 & 0.640 \\
\hline DPL-Corr & 0.709 & 0.767 & 0.600 & 0.673 \\
\hline FASpell & 0.742 & 0.676 & 0.600 & 0.635 \\
\hline SpellGCN $\dagger$ & - & 0.710 & 0.640 & 0.673 \\
\hline MERT & 0.768 & 0.881 & 0.620 & 0.728 \\
\hline BERT & 0.763 & 0.849 & 0.641 & 0.730 \\
\hline FixedFilt & 0.757 & 0.900 & 0.580 & 0.705 \\
\hline GlyceFilt & 0.764 & 0.902 & 0.592 & 0.715 \\
\hline HeadFilt & $\begin{array}{r}\mathbf{0 . 7 7 3} \\
\text { TABI }\end{array}$ & $\begin{array}{l}0.894 \\
\text { E III }\end{array}$ & 0.619 & 0.731 \\
\hline
\end{tabular}

Traditional Chinese error prediction. The means of 5 runs with different seeds are reported. See Section IV-B for list of baselines. Acc: Accuracy, Pre: Precision, Rec: Recall

is the current SOTA for many different Chinese NLP tasks and is therefore a strong baseline. Results of winning teams of the Bake-offs are also included: [56] (SMT), [14] (n-gram LM), National Kaohsiung University of Applied Sciences (KUAS), [30] (HanSpeller), as well as recent results by [24] (DPLCorr), [33] (FASpell), and [29] (MERT).

Note that Wang et al. [57] and Cheng et al. [34] used different training and test sets, different evaluation tools, and Simplified Chinese characters instead of the original Traditional Chinese. Therefore their results are not directly comparable to previously mentioned studies. We include our best attempt to compare with [34,57] in Section IV-E.

\section{Experimental Setup}

We used tools provided by the CSC Bake-offs [3] to evaluate accuracy, precision, recall, and F1 score. Metrics are calculated at the sentence level, i.e. a prediction is correct if all errors in the sentence are predicted correctly.

The models are implemented using PyTorch [58]. BERT model is trained using cross-entropy loss for 20 epochs using a learning rate of $3 e^{-5}$ and a batch size of 16 with AdamW [59]. We repeated this training 5 times using 5 different random seeds and reported the average. HeadFilt is trained using the contrastive loss [48] with Adam [60]. We used a learning rate of $3 e^{-3}$ and a batch size of 500. The hierarchical

\begin{tabular}{|c|c|c|c|c|}
\hline Correction & Acc. & Pre. & Rec. & F1 \\
\hline \multicolumn{5}{|c|}{2013 Bake-off } \\
\hline SMT & 0.443 & 0.699 & - & - \\
\hline n-gram LM & 0.625 & 0.703 & - & - \\
\hline FASSpell & 0.605 & 0.731 & - & - \\
\hline BERT & 0.489 & 0.597 & - & - \\
\hline FixedFilt & 0.575 & 0.737 & - & - \\
\hline GlyceFilt & 0.537 & 0.712 & - & - \\
\hline HeadFilt & 0.591 & 0.754 & - & - \\
\hline \multicolumn{5}{|c|}{2014 Bake-off } \\
\hline KUAS & 0.708 & 0.910 & 0.461 & 0.612 \\
\hline FASpell & 0.693 & 0.594 & 0.520 & 0.554 \\
\hline SpellGCN $\dagger$ & - & 0.510 & 0.477 & 0.493 \\
\hline MERT & 0.681 & 0.774 & 0.510 & 0.615 \\
\hline BERT & 0.650 & 0.740 & 0.464 & 0.570 \\
\hline FixedFilt & 0.684 & 0.816 & 0.475 & 0.601 \\
\hline GlyceFilt & 0.681 & 0.811 & 0.472 & 0.597 \\
\hline HeadFilt & 0.698 & 0.811 & 0.517 & 0.631 \\
\hline \multicolumn{5}{|c|}{2015 Bake-off } \\
\hline HanSpeller & 0.691 & 0.797 & 0.514 & 0.625 \\
\hline DPL-Corr & 0.695 & 0.759 & 0.572 & 0.652 \\
\hline FASpell & 0.737 & 0.666 & 0.591 & 0.626 \\
\hline SpellGCN $\dagger$ & - & 0.601 & 0.542 & 0.570 \\
\hline MERT & 0.746 & 0.873 & 0.576 & 0.694 \\
\hline BERT & 0.689 & 0.812 & 0.492 & 0.613 \\
\hline FixedFilt & 0.729 & 0.890 & 0.523 & 0.658 \\
\hline GlyceFilt & 0.730 & 0.891 & 0.525 & 0.661 \\
\hline HeadFilt & 0.746 & 0.885 & 0.565 & 0.690 \\
\hline
\end{tabular}

Traditional Chinese error correction. The means of 5 runs with different seeds are reported. $\dagger$ Results reported by Bao et al. [29].

embeddings' dimension is 512. HeadFilt is trained in two steps (see Section [II-B). HeadFilt is first trained to imitate the given confusion sets for 150k steps. HeadFilt is then further trained using the additional error pairs observed in the training data for $50 \mathrm{k}$ steps.

\section{Results for Traditional Chinese Data}

For error detection (Table III), approaches with filtering (FixedFilt, GlyceFilt, and HeadFilt) achieved higher precision and lower recall when compared to the non-filtering baseline (BERT). HeadFilt was consistently better than the BERT baseline in terms of the accuracy and F1 score, validating the benefit of filtering. HeadFilt was also consistently better than FixedFilt, showing that adaptation is beneficial. HeadFilt was as good as if not better than GlyceFilt, suggesting that the inductive bias of hierarchical embeddings is suitable for filtering. The proposed HeadFilt model also achieves SOTA results for error prediction F1 score for 2014 and 2015.

For error correction (Table IV], HeadFilt is better than FixedFilt and BERT in all datasets in both accuracy and F1 score. Whereas filtering lowers recall in error prediction, it leads to higher recall in error correction since filtering lowers the number of correction candidates to be considered. HeadFilt achieved consistently higher recall than the FixedFilt baseline. HeadFilt is also better than the SOTA result for F1 score in 2014 and 2015. 


\begin{tabular}{|c|c|c|c|c|}
\hline & Acc. & Pre. & Rec. & $\mathrm{F} 1$ \\
\hline \multicolumn{5}{|c|}{2013 Bake-off } \\
\hline SpellGCN & - & 0.801 & 0.744 & 0.772 \\
\hline BERT & 0.742 & 1.000 & 0.742 & 0.852 \\
\hline FixedFilt & 0.732 & 1.000 & 0.732 & 0.845 \\
\hline GlyceFilt & 0.749 & 1.000 & 0.749 & 0.856 \\
\hline HeadFilt & 0.749 & 1.000 & 0.749 & 0.857 \\
\hline \multicolumn{5}{|c|}{2014 Bake-off } \\
\hline SpellGCN & - & 0.651 & 0.695 & 0.672 \\
\hline BERT & 0.733 & 0.800 & 0.621 & 0.699 \\
\hline FixedFilt & 0.712 & 0.815 & 0.550 & 0.657 \\
\hline GlyceFilt & 0.707 & 0.833 & 0.518 & 0.639 \\
\hline HeadFilt & 0.742 & 0.825 & 0.616 & 0.705 \\
\hline \multicolumn{5}{|c|}{2015 Bake-off } \\
\hline SpellGCN & - & 0.748 & 0.807 & 0.777 \\
\hline BERT & 0.785 & 0.827 & 0.721 & 0.770 \\
\hline FixedFilt & 0.766 & 0.844 & 0.653 & 0.736 \\
\hline GlyceFilt & 0.781 & 0.871 & 0.661 & 0.752 \\
\hline HeadFilt & 0.793 & 0.845 & 0.718 & 0.776 \\
\hline
\end{tabular}

\section{E. Results for Simplified Chinese Data}

Due to differences in the experimental setup, results from Wang et al. [57] and Cheng et al. [34] are not directly comparable to those from prior studies [24]33]. First, datasets were converted from Traditional to Simplified Chinese in [34, 57], potentially altering the results. This is because distinct traditional characters could be merged and replaced with the same Simplified character, so a character may be correct in Simplified Chinese but erroneous in Traditional Chinese [33]. For example, the character 周 in 周末 (“weekend") is correct in Simplified Chinese, but it is an error in Traditional Chinese (the correct character is 週). Second, Wang et al. [57] evaluated only on sentences with errors from the original test sets using character level metrics instead of evaluating all the test set sentences using sentence level metrics as it was done at the Bake-offs. Since we are following the standard set up from the Bake-offs, it is tricky to compare fairly with [57].

However, it is possible to compare with Cheng et al. [34]'s result since they also evaluated using sentence level metrics. We attempted to compare against Cheng et al. [34]'s method (SpellGCN) by training our models with their training data and evaluated on their test data (in Simplified Chinese). The results in Table $\mathrm{V}$ and $\mathrm{VI}$ show that HeadFilt outperforms SpellGCN in all three years. HeadFilt was also generally better than GlyceFilt. Interestingly, the FixedFilt baseline is worse than the baseline without filtering (i.e. BERT). The confusion sets used in the FixedFilt baseline are obtained by converting the original confusion sets into Simplified script. The drop in performance of FixedFilt could be because the confusion sets in simplified scripts do not capture the errors well. In this

\begin{tabular}{|c|c|c|c|c|}
\hline & Acc. & Pre. & Rec. & $\mathrm{F} 1$ \\
\hline \multicolumn{5}{|c|}{2013 Bake-off } \\
\hline SpellGCN & - & 0.783 & 0.727 & 0.754 \\
\hline BERT & 0.722 & 1.000 & 0.722 & 0.839 \\
\hline FixedFilt & 0.709 & 1.000 & 0.709 & 0.829 \\
\hline GlyceFilt & 0.739 & 1.000 & 0.739 & 0.850 \\
\hline HeadFilt & 0.741 & 1.000 & 0.741 & 0.851 \\
\hline \multicolumn{5}{|c|}{2014 Bake-off } \\
\hline SpellGCN & - & 0.631 & 0.672 & 0.653 \\
\hline BERT & 0.721 & 0.794 & 0.597 & 0.681 \\
\hline FixedFilt & 0.699 & 0.807 & 0.524 & 0.635 \\
\hline GlyceFilt & 0.700 & 0.830 & 0.504 & 0.627 \\
\hline HeadFilt & 0.735 & 0.821 & 0.602 & 0.694 \\
\hline \multicolumn{5}{|c|}{2015 Bake-off } \\
\hline SpellGCN & - & 0.721 & 0.777 & 0.759 \\
\hline BERT & 0.770 & 0.821 & 0.691 & 0.751 \\
\hline FixedFilt & 0.745 & 0.835 & 0.610 & 0.705 \\
\hline GlyceFilt & 0.773 & 0.868 & 0.644 & 0.740 \\
\hline HeadFilt & $\mathbf{0 . 7 8 5}$ & 0.842 & 0.702 & 0.765 \\
\hline
\end{tabular}

Simplified Chinese error correction. The means of 5 runs with different seeds are reported.

setup using simplified characters, only $60 \%$ of the errors in the training set are covered by the confusion sets as compared to more than $75 \%$ in the setup using traditional characters (Table II). Such a large shift in error domains severely affects model performance of FixedFilt. In contrast, with domain adaptation, the HeadFilt model still performs well. Comparing Table III] and IV with Table V and VI shows how model performance varies across different experimental setups.

\section{F. Ablation Study}

We perform ablation to gauge the contribution of components to the performance of HeadFilt. We compared the full HeadFilt model against the HeadFilt model without domain adaptation in Section IV-F1. This is denoted as "-Ad" in Table VII and VIII We further assessed the contribution from the hierarchical embeddings in Section IV-F2 by replacing hierarchical embeddings with standard embeddings. This is denoted as "-Ad -HE".

1) Adaptation Analysis: Domain adaptation contributed little to HeadFilt performance in 2013 since removing this step resulted in the same accuracy and F1 score for both error prediction and correction. This is not surprising considering that $93.68 \%$ of the errors in the training data is already covered by the confusion sets (shown in Table III), therefore, adaptation only add 17 new pairs of positive examples. For 2014 and 2015, domain adaptation shows more tangible contribution, especially for error correction. This result aligns with statistics in Table II. which shows larger domain-shifts in 2014 and 2015. 


\begin{tabular}{lcccc} 
Prediction & Acc. & Pre. & Rec. & $F 1$ \\
\hline \multicolumn{5}{c}{2013 Bake-off } \\
\hline HeadFilt & 0.914 & 0.891 & 0.813 & 0.850 \\
-Ad & $\mathbf{0 . 9 1 5}$ & 0.894 & 0.813 & $\mathbf{0 . 8 5 1}$ \\
-Ad -HE & 0.911 & 0.906 & 0.787 & 0.842 \\
FixedFilt & 0.910 & 0.884 & 0.804 & 0.842 \\
\hline \multicolumn{5}{c}{2014 Bake-off } \\
\hline HeadFilt & $\mathbf{0 . 7 1 9}$ & 0.823 & 0.559 & $\mathbf{0 . 6 6 6}$ \\
-Ad & 0.717 & 0.827 & 0.548 & 0.659 \\
-Ad -HE & 0.714 & 0.826 & 0.542 & 0.654 \\
FixedFilt & 0.709 & 0.831 & 0.526 & 0.644 \\
\hline \multicolumn{5}{c}{2015 Bake-off } \\
\hline HeadFilt & $\mathbf{0 . 7 7 3}$ & 0.894 & 0.619 & $\mathbf{0 . 7 3 1}$ \\
-Ad & $\mathbf{0 . 7 7 3}$ & 0.903 & 0.611 & 0.729 \\
-Ad -HE & 0.765 & 0.906 & 0.592 & 0.716 \\
FixedFilt & 0.757 & 0.900 & 0.580 & 0.705
\end{tabular}

Traditional Chinese error prediction ablation. The means of 5 runs with different seeds are reported. Ad: Adaptation, HE: hierarchical embedding,

2) Hierarchical Embedding Analysis: Besides domain adaptation, the use of hierarchical embeddings also contributed to the performance gain since replacing hierarchical with standard embeddings leads to worse performance for both error prediction and correction. This trend is observed in all 3 datasets. Besides, HeadFilt without domain adaptation ("Ad") consistently beats FixedFilt, demonstrating the benefit of hierarchical embeddings. Hierarchical embeddings impose additional constraints (inductive bias) on the embedding space, such that characters with similar morphology are more likely to have similar character embeddings. This inductive bias could make it more effective to automatically learn which sets of characters are more likely to be mistaken from one another (See Section $\mathrm{V}-\mathrm{A}$.

\section{DISCUSSION}

\section{A. Hierarchical Embeddings Enables Automated Domain Adaptation}

Section IV-E shows that using confusion sets without sufficient coverage could hurt performance. Ideally, there should be a confusion set of at least 100 candidates for every character [11]. The confusion sets used in this work [61] cover only 5,401 high frequency Traditional Chinese characters and only $30 \%$ of the confusion sets have more than 100 candidates. Filtering using these fixed confusion sets could miss many spelling errors, especially for text from other domains (e.g. Simplified Chinese text or technical documents). Changes in word usage might also make confusion sets obsolete since past spelling errors could become widely accepted and used and are no longer considered as errors. For example, in English, "ingot" was a spelling error of the word "lingot". However, as the usage of "ingot" spread, "ingot" became the dominant spelling and is considered a correct word nowadays.

HeadFilt is applicable to text in different domains or to text written by different demographic groups because of its adaptability. Theoretically, one could construct confusion sets automatically [32, 62, 63] for all characters. In practice, it is

\begin{tabular}{lllll} 
Correction & Acc. & Pre. & Rec. & F1 \\
\hline \multicolumn{5}{c}{2013 Bake-off } \\
\hline HeadFilt & $\mathbf{0 . 5 9 1}$ & 0.754 & - & - \\
-Ad & 0.590 & 0.753 & - & - \\
-Ad -HE & 0.577 & 0.755 & - & - \\
FixedFilt & 0.575 & 0.737 & - & - \\
\hline \multicolumn{5}{c}{2014 Bake-off } \\
\hline HeadFilt & $\mathbf{0 . 6 9 8}$ & 0.811 & 0.517 & $\mathbf{0 . 6 3 1}$ \\
-Ad & 0.692 & 0.813 & 0.498 & 0.617 \\
-Ad -HE & 0.691 & 0.813 & 0.496 & 0.616 \\
FixedFilt & 0.684 & 0.816 & 0.475 & 0.601 \\
\hline \multicolumn{5}{c}{2015 Bake-off } \\
\hline HeadFilt & $\mathbf{0 . 7 4 6}$ & 0.885 & 0.565 & $\mathbf{0 . 6 9 0}$ \\
-Ad & 0.743 & 0.894 & 0.552 & 0.683 \\
-Ad -HE & 0.742 & 0.899 & 0.546 & 0.679 \\
FixedFilt & 0.729 & 0.890 & 0.523 & 0.658 \\
& TABLE VIII
\end{tabular}

Traditional Chinese error correction ablation. The means of 5 runs with different seeds are reported.

difficult to construct ones that work well across different input methods (typed and written), different demographics (including new populations with specific slangs and vocabulary) and different domains. Constructed confusion sets eventually get outdated overtime, so domain adaptation approaches are useful for a number of reasons.

Domain adaptation for filters requires estimating similarity between Chinese characters, which is algorithmically challenging because Chinese characters are not sequences of sub-character units; they have hierarchical structures. Previous work estimated similarity between characters using handcrafted formulas based on edit distance [33, 64], Dice coefficients [11], or overlap in constituent structure [65]. Edit distance was originally formulated for sequences while Dice coefficient was originally formulated for sets, so they might not capture the hierarchical nature of Chinese characters well. Besides, since these measures are handcrafted, they likely require manual fine-tuning to work well. In contrast, hierarchical embeddings can better characterize the similarity between Chinese characters and enable automated domain adaptation, reducing the manual effort to keep the filters upto-date.

\section{B. Better Recall with HeadFilt}

The way hierarchical embeddings are optimized (Figure 6 could conceivably explain why HeadFilt achieves higher error correction recall than filtering using given confusion sets (FixedFilt) (Section IV-D and IV-F). As 吾 is a substitution error of 無, minimizing the contrastive loss increases their similarity and reduces the L2 distance between their embeddings. Since 無 and 舞 have similar morphological structures, their hierarchical embeddings would be close in the embedding space. Thus, increasing the similarity between 吾 and 無 would indirectly increase the similarity between 吾 and 舞. Even though 吾 and 舞 are not observed as substitution errors, by leveraging the morphological similarity, HeadFilt can still infer that there might be a substitution error. Since many 


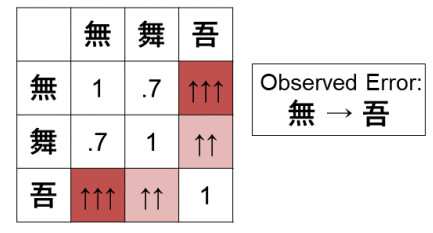

Fig. 6. Learning similarity without observation. Arrows indicate magnitude of change. Increasing the similarity score between 吾 and 無 indirectly increases the similarity score between 吾 and 舞.

errors are not observed during training, using hierarchical embeddings widens the filter's coverage.

\section{Future Work}

Although HeadFilt models characters' similarity using their structures, which implicitly encode pronunciation [10, 66], there may still be ambiguous cases. Therefore, incorporating Pinyin or Zhuyin into the proposed HeadFilt could be a possible extension that further enhances similarity estimation and consequently improves spell checking. This is an interesting possibility for future work, as the design and construction of such a study would require one to explicitly consider the contexts of the various different phonetic and tonal representations of a heteronym (a heteronym is a Chinese character that can take on multiple pronunciations), which requires extensive linguistic expertise and resources. Such efforts are out of the scope of this current endeavor.

Although this work focuses on spell check for Chinese, it can be extended to languages with similar logographic and phonological structures such as Japanese, Korean, or Vietnamese [67,68]. Besides spell check, other tasks such as automatic speech recognition may also achieve higher recall by using HeadFilt. There are many homophones in Chinese languages and predicting the correct characters from the audio signal is at times challenging. HeadFilt can narrow the list of candidate characters with similar pronunciation to lower the perplexity for speech recognition.

\section{CONCLUSION}

We presented a filtering model using hierarchical character embeddings (HeadFilt) for Chinese spell check. Our approach achieved SOTA error correction results on two spell check datasets. The adaptability of HeadFilt makes it possible to tailor proposed model to text from different domains, text written using different input methods, and potentially text written by different individuals.

\section{REFERENCES}

[1] S.-H. Wu, C.-L. Liu, and L.-H. Lee, "Chinese spelling check evaluation at SIGHAN Bake-off 2013," in Proceedings of the Seventh SIGHAN Workshop on Chinese Language Processing, 2013.

[2] L.-C. Yu, L.-H. Lee, Y.-H. Tseng, and H.-H. Chen, "Overview of SIGHAN 2014 bake-off for Chinese spelling check," in Proceedings of The Third CIPS-SIGHAN Joint Conference on Chinese Language Processing, 2014.

[3] Y.-H. Tseng, L.-H. Lee, L.-P. Chang, and H.-H. Chen, "Introduction to SIGHAN 2015 bake-off for Chinese spelling check," in Proceedings of the Eighth SIGHAN Workshop on Chinese Language Processing, 2015.
[4] S. Ben-David, J. Blitzer, K. Crammer, and F. Pereira, "Analysis of representations for domain adaptation," in Advances in neural information processing systems, 2007, pp. 137-144.

[5] J. Quionero-Candela, M. Sugiyama, A. Schwaighofer, and N. D. Lawrence, Dataset shift in machine learning, 1st ed. The MIT Press, 2009.

[6] S.-H. Wu, Y.-Z. Chen, P.-c. Yang, T. Ku, and C.-L. Liu, "Reducing the false alarm rate of Chinese character error detection and correction," in CIPS-SIGHAN Joint Conference on Chinese Language Processing, 2010.

[7] C.-H. Wang, J. S. Chang, and J.-C. Wu, "Automatic chinese confusion words extraction using conditional random fields and the web," in Proceedings of the Seventh SIGHAN Workshop on Chinese Language Processing, 2013, pp. 64-68.

[8] W.-C. Chu and C.-J. Lin, "Ntou chinese spelling check system in clp bake-off 2014," in Proceedings of The Third CIPS-SIGHAN Joint Conference on Chinese Language Processing, 2014, pp. 210-215.

[9] W. Xie, P. Huang, X. Zhang, K. Hong, Q. Huang, B. Chen, and L. Huang, "Chinese spelling check system based on n-gram model," in Proceedings of the Eighth SIGHAN Workshop on Chinese Language Processing, 2015.

[10] M. Nguyen, H. G. Ngo, and N. F. Chen, "Hierarchical character embeddings: Learning phonological and semantic representations in languages of logographic origin using recursive neural networks," IEEE/ACM Transactions on Audio, Speech, and Language Processing, 2020.

[11] C.-L. Liu, M.-H. Lai, K.-W. Tien, Y.-H. Chuang, S.-H. Wu, and C.-Y. Lee, "Visually and phonologically similar characters in incorrect Chinese words: Analyses, identification, and applications," ACM Transactions on Asian Language Information Processing (TALIP), vol. 10, no. 2, 2011.

[12] Y. Meng, W. Wu, F. Wang, X. Li, P. Nie, F. Yin, M. Li, Q. Han, X. Sun, and J. Li, "Glyce: Glyph-vectors for chinese character representations," in Advances in Neural Information Processing Systems, 2019, pp. 27462757.

[13] C.-H. Chang, "A new approach for automatic Chinese spelling correction," in Proceedings of Natural Language Processing Pacific Rim Symposium, vol. 95, 1995.

[14] J.-F. Yeh, S.-F. Li, M.-R. Wu, W.-Y. Chen, and M.-C. Su, "Chinese word spelling correction based on n-gram ranked inverted index list," in Proceedings of the Seventh SIGHAN Workshop on Chinese Language Processing, 2013, pp. 43-48.

[15] Q. Huang, P. Huang, X. Zhang, W. Xie, K. Hong, B. Chen, and L. Huang, "Chinese spelling check system based on tri-gram model," in Proceedings of the third CIPS-SIGHAN joint conference on Chinese language processing, 2014, pp. 173-178.

[16] Y.-R. Wang and Y.-F. Liao, "Nctu and ntut's entry to clp-2014 chinese spelling check evaluation," in Proceedings of The Third CIPS-SIGHAN Joint Conference on Chinese Language Processing, 2014, pp. 216-219.

[17] L. Gu, Y. Wang, and X. Liang, "Introduction to njupt chinese spelling check systems in clp-2014 bakeoff," in Proceedings of The Third CIPS SIGHAN Joint Conference on Chinese Language Processing, 2014, pp. 167-172.

[18] Z. Jia, P. Wang, and H. Zhao, "Graph model for chinese spell checking," in Proceedings of the Seventh SIGHAN Workshop on Chinese Language Processing, 2013, pp. 88-92.

[19] Z. Jia and H. Zhao, "A joint graph model for pinyin-to-Chinese conversion with typo correction," in Proceedings of ACL, 2014.

[20] Y. Xin, H. Zhao, Y. Wang, and Z. Jia, "An improved graph model for chinese spell checking," in Proceedings of The Third CIPS-SIGHAN Joint Conference on Chinese Language Processing, 2014, pp. 157-166.

[21] H. Zhao, D. Cai, Y. Xin, Y. Wang, and Z. Jia, "A hybrid model for Chinese spelling check," ACM Transactions on Asian and Low-Resource Language Information Processing (TALLIP), vol. 16, no. 3, 2017.

[22] J. Xiong, Q. Zhang, J. Hou, Q. Wang, Y. Wang, and X. Cheng, "Extended hmm and ranking models for chinese spelling correction," in Proceedings of The Third CIPS-SIGHAN Joint Conference on Chinese Language Processing, 2014, pp. 133-138.

[23] J. Duan, B. Wang, Z. Tan, X. Wei, and H. Wang, "Chinese spelling check via bidirectional 1stm-crf," in 2019 IEEE 8th Joint International Information Technology and Artificial Intelligence Conference (ITAIC). IEEE, 2019, pp. 1333-1336.

[24] H. Xie, A. Li, Y. Li, J. Cheng, Z. Chen, X. Lyu, and Z. Tang, "Automatic Chinese Spelling Checking and Correction Based on CharacterBased Pre-trained Contextual Representations," in CCF International Conference on Natural Language Processing and Chinese Computing. Springer, 2019. 
[25] L.-C. Yu, C.-H. Liu, and C.-H. Wu, "Candidate scoring using web-based measure for Chinese spelling error correction," in Proceedings of the Seventh SIGHAN Workshop on Chinese Language Processing, 2013.

[26] C.-W. Li, J.-J. Chen, and J. S. Chang, "Chinese spelling check based on neural machine translation," in Proceedings of the 32nd Pacific Asia Conference on Language, Information and Computation, 2018.

[27] Y.-M. Hsieh, M.-H. Bai, and K.-J. Chen, "Introduction to CKIP Chinese spelling check system for SIGHAN Bakeoff 2013 evaluation," in Proceedings of the Seventh SIGHAN Workshop on Chinese Language Processing, 2013.

[28] J. Yu and Z. Li, "Chinese spelling error detection and correction based on language model, pronunciation, and shape," in Proceedings of The Third CIPS-SIGHAN Joint Conference on Chinese Language Processing, 2014.

[29] Z. Bao, C. Li, and R. Wang, "Chunk-based chinese spelling check with global optimization," in Proceedings of EMNLP: Findings, 2020, pp. 2031-2040. [Online]. Available: https://www.aclweb.org/anthology/ 2020.findings-emnlp.184

[30] S. Zhang, J. Xiong, J. Hou, Q. Zhang, and X. Cheng, "Hanspeller++: A unified framework for chinese spelling correction," in Proceedings of the Eighth SIGHAN Workshop on Chinese Language Processing, 2015, pp. 38-45.

[31] X. Liu, K. Cheng, Y. Luo, K. Duh, and Y. Matsumoto, "A hybrid Chinese spelling correction using language model and statistical machine translation with reranking," in Proceedings of the Seventh SIGHAN Workshop on Chinese Language Processing, 2013.

[32] D. Wang, Y. Song, J. Li, J. Han, and H. Zhang, "A hybrid approach to automatic corpus generation for Chinese spelling check," in Proceedings of EMNLP, 2018.

[33] Y. Hong, X. Yu, N. He, N. Liu, and J. Liu, "Faspell: A fast, adaptable, simple, powerful chinese spell checker based on dae-decoder paradigm," in Proceedings of the 5th Workshop on Noisy User-generated Text (WNUT 2019), 2019, pp. 160-169.

[34] X. Cheng, W. Xu, K. Chen, S. Jiang, F. Wang, T. Wang, W. Chu, and Y. Qi, "Spellgen: Incorporating phonological and visual similarities into language models for chinese spelling check," in Proceedings of ACL, 2020.

[35] H. Daumé III, "Frustratingly Easy Domain Adaptation," in Proceedings of ACL, 2007, pp. 256-263. [Online]. Available: https://www.aclweb. org/anthology/P07-1033

[36] X. Glorot, A. Bordes, and Y. Bengio, "Domain adaptation for large-scale sentiment classification: A deep learning approach," in Proceedings of ICML, 2011.

[37] T. Schnabel and H. Schütze, "Flors: Fast and simple domain adaptation for part-of-speech tagging," Transactions of the Association for Computational Linguistics, vol. 2, pp. 15-26, 2014.

[38] J. Blitzer, R. McDonald, and F. Pereira, "Domain adaptation with structural correspondence learning," in Proceedings of the 2006 conference on empirical methods in natural language processing, 2006, pp. 120-128. [Online]. Available: https://www.aclweb.org/anthology/ W06-1615

[39] M. Dredze, J. Blitzer, P. Talukdar, K. Ganchev, J. Graca, and F. Pereira, "Frustratingly hard domain adaptation for dependency parsing," in Proceedings of EMNLP, 2007, pp. 1051-1055. [Online]. Available: https://www.aclweb.org/anthology/D07-1112

[40] S. Ruder and B. Plank, "Strong baselines for neural semi-supervised learning under domain shift," in Proceedings of ACL, 2018, pp. 1044 1054.

[41] J. Devlin, M.-W. Chang, K. Lee, and K. Toutanova, "BERT: Pretraining of deep bidirectional transformers for language understanding," in Proceedings of NAACL-HLT, 2019.

[42] P. Ramachandran, P. Liu, and Q. Le, "Unsupervised pretraining for sequence to sequence learning," in Proceedings of EMNLP, 2017.

[43] M. E. Peters, M. Neumann, M. Iyyer, M. Gardner, C. Clark, K. Lee, and L. Zettlemoyer, "Deep contextualized word representations," in Proceedings of NAACL-HLT, 2018.

[44] A. Radford, K. Narasimhan, T. Salimans, and I. Sutskever, "Improving language understanding by generative pre-training," 2018.

[45] J. Howard and S. Ruder, "Universal language model fine-tuning for text classification," in Proceedings of ACL, 2018.

[46] K. S. Tai, R. Socher, and C. Manning, "Improved semantic representations from tree-structured long short-term memory networks," in Proceedings of ACL, 2015.

[47] X. Zhu, P. Sobihani, and H. Guo, "Long short-term memory over recursive structures," in Proceedings of ICML, 2015.
[48] R. Hadsell, S. Chopra, and Y. LeCun, "Dimensionality reduction by learning an invariant mapping," in Proceedings of $C V P R$, vol. 2. IEEE, 2006, pp. 1735-1742.

[49] X. Shi, J. Zhai, X. Yang, Z. Xie, and C. Liu, "Radical embedding: Delving deeper to Chinese radicals," in Proceedings of ACL, vol. 2, 2015.

[50] R. Yin, Q. Wang, P. Li, R. Li, and B. Wang, "Multi-granularity Chinese word embedding," in Proceedings of EMNLP, 2016.

[51] F. Liu, H. Lu, C. Lo, and G. Neubig, "Learning character-level compositionality with visual features," in Proceedings of ACL, 2017, pp. 2059-2068.

[52] J. Yu, X. Jian, H. Xin, and Y. Song, "Joint embeddings of chinese words, characters, and fine-grained subcharacter components," in Proceedings of EMNLP, 2017.

[53] T.-R. Su and H.-Y. Lee, "Learning Chinese word representations from glyphs of characters," in Proceedings of EMNLP, 2017.

[54] S. Cao, W. Lu, J. Zhou, and X. Li, "cw2vec: Learning Chinese word embeddings with stroke n-grams," in Proceedings of AAAI, 2018, pp. 5053-5061

[55] H. Zhuang, C. Wang, C. Li, Y. Li, Q. Wang, and X. Zhou, "Chinese language processing based on stroke representation and multidimensional representation," IEEE Access, vol. 6, pp. 41 928-41941, 2018.

[56] H.-w. Chiu, J.-c. Wu, and J. S. Chang, "Chinese spelling checker based on statistical machine translation," in Proceedings of the Seventh SIGHAN Workshop on Chinese Language Processing, 2013, pp. 49-53.

[57] D. Wang, Y. Tay, and L. Zhong, "Confusionset-guided pointer networks for Chinese spelling check," in Proceedings of ACL, 2019.

[58] A. Paszke, S. Gross, F. Massa, A. Lerer, J. Bradbury, G. Chanan, T. Killeen, Z. Lin, N. Gimelshein, L. Antiga et al., "Pytorch: An imperative style, high-performance deep learning library," in Proceedings of NeurIPS, 2019, pp. 8026-8037.

[59] I. Loshchilov and F. Hutter, "Decoupled weight decay regularization," in Proceedings of ICLR, 2018.

[60] D. P. Kingma and J. Ba, "Adam: A Method for Stochastic Optimization," in Proceedings of ICLR, 2014.

[61] C.-L. Liu, K.-W. Tien, M.-H. Lai, Y.-H. Chuang, and S.-H. Wu, "Phonological and logographic influences on errors in written Chinese words," in Proceedings of the 7th Workshop on Asian Language Resources, 2009.

[62] B. Chen, "Word topic models for spoken document retrieval and transcription," ACM Transactions on Asian Language Information Processing (TALIP), vol. 8, no. 1, p. 2, 2009.

[63] H.-w. Chiu, J.-c. Wu, and J. S. Chang, "Chinese spell checking based on noisy channel model," in Proceedings of The Third CIPS-SIGHAN Joint Conference on Chinese Language Processing, 2014, pp. 202-209.

[64] K.-Y. Chen, H.-S. Lee, C.-H. Lee, H.-M. Wang, and H.-H. Chen, "A study of language modeling for Chinese spelling check," in Proceedings of the Seventh SIGHAN Workshop on Chinese Language Processing, 2013.

[65] T.-H. Chang, H.-C. Chen, Y.-H. Tseng, and J.-L. Zheng, "Automatic detection and correction for Chinese misspelled words using phonological and orthographic similarities," in Proceedings of the Seventh SIGHAN Workshop on Chinese Language Processing, 2013.

[66] M. Nguyen, H. G. Ngo, and N. F. Chen, "Multimodal neural pronunciation modeling for spoken languages with logographic origin," in Proceedings of EMNLP, 2018.

[67] H. G. Ngo, N. F. Chen, S. Sivadas, B. Ma, and H. Li, "A MinimalResource Transliteration Framework for Vietnamese," in Proceedings of Interspeech, 2014

[68] H. G. Ngo, M. Nguyen, and N. F. Chen, "Phonology-augmented statistical framework for machine transliteration using limited linguistic resources," IEEE/ACM Transactions on Audio, Speech and Language Processing, vol. 27, no. 1, 2019. 